\title{
Ciencia e ideología. La polémica de 1975 entre G. Kli- movsky, O. Varsavsky y T. Moro Simpson
}

\begin{abstract}
Resumen: El presente trabajo analiza las posiciones asumidas por Gregorio Klimovsky, Oscar Varsavsky y Thomas Moro Simpson en el debate que sostuvieron sobre ciencia e ideología, recopilado en 1975 en un libro y en diversos artículos publicados por la revista Ciencia Nueva. Se analizan específicamente sus señalamientos sobre la objetividad en la ciencia, la relación entre ciencia y sociedad, el rol del científico en la sociedad y la relación entre actividad científica y política. Se destaca la originalidad que presenta el debate en términos de la relación entre epistemología, ciencia, política y procesos sociales, que permite repensar los aportes de los autores en torno a la actualidad del Pensamiento Latinoamericano en Ciencia, Tecnología y Desarrollo (PLACTED) y del campo CTS.
\end{abstract}

Palabras clave: Revista Ciencia Nueva, Epistemología, Política, Sociedad, Klimovsky, Gregorio, Varsavsky, Oscar, Moro Simpson, Thomas.

\section{Science and ideology. The 1975 controversy between G. Klimovsky, O. Varsavsky and T. Moro Simpson}

\begin{abstract}
This paper analyzes the positions assumed by Gregorio Klimovsky, Oscar Varsavsky and Thomas Moro Simpson in the debate they held on science and ideology, compiled in 1975 in a book and in various articles published by the journal Ciencia Nueva. His remarks on objectivity in science, the relationship between science and society, the role of the scientist in society, and the relationship between scientific and political activity are specifically analyzed. The originality presented by the debate in terms of the relationship between epistemology, science, politics and social processes is highlighted, which allows us to rethink the contributions of the authors regarding the current situation of Latin American Thought in Science, Technology and Development (PLACTED) and the CTS field.
\end{abstract}

Keywords: New Science Magazine, Science, Epistemology, Politics, Society, Klimovsky, Gregorio, Varsavsky, Oscar, Moro Simpson, Thomas

\section{Ciência e ideologia. A controvérsia de 1975 entre G. Klimovsky, O. Varsavsky e T. Moro Simpson}

Resumo: Este artigo analisa as posições assumidas por Gregorio Klimovsky, Oscar Varsavsky e Thomas Moro Simpson no debate que travaram sobre ciência e ideologia, compilado, em 1975, em um livro e em vários artigos publicados pela revista Ciencia Nueva. Suas observações sobre a objetividade na ciência, a relação entre ciência e sociedade, o papel do cientista na sociedade e a relação entre a atividade científica e política são analisadas especificamente. Ressalta-se a originalidade apresentada pelo debate em termos da relação entre epistemologia, ciência, política e processos sociais, o que nos permite repensar as contribuições dos autores a respeito da situação atual do Pensamento Latino-Americano em Ciência, Tecnologia e Desenvolvimento (PLACTED) e o campo CTS.

Palavras-chave: Revista New Science, Ciência, Epistemologia, Política, Sociedade, Klimovsky, Gregorio, Varsavsky, Oscar, Moro Simpson, Thomas

\section{Ciencia Tecnología y Política \\ Año $4 \mathrm{~N}^{\circ} 7$ Noviembre 2021}

Ezequiel Asprella

Universidad Nacional de Tres de Febrero y Universidad

Nacional de La Plata asprella.ezequiel@gmail.com

Año $4 \mathrm{~N}^{\circ} 7$ Noviembre 2021

Fecha de recibido: 31/07/2021

Fecha de aprobado: 19/10/2021

https://doi.org/10.24215/26183188e067 https://revistas.un|p.edu.ar/CTyP ISSN 2618-3188

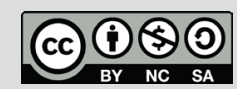

Esta obra está bajo licencia Creative Commons Atribución-NoComercial-Compartirlgual 4.0 Internacional http://creativecommons.org/licenses/bync-sa/4.O/deed.es_AR 


\section{Ciencia e ideología. La polémica de 1975 entre G. Klimovsky, O. Varsavsky y T. Moro}

\section{Ezequiel Asprella.}

Universidad Nacional de Tres de Febrero y Universidad Nacional de La Plata asprella.ezequie/@gmail.com

Resumen: El presente trabajo analiza las posiciones asumidas por Gregorio Klimovsky, Oscar Varsavsky y Thomas Moro Simpson en el debate que sostuvieron sobre ciencia e ideología, recopilado en 1975 en un libro y en diversos artículos publicados por la revista Ciencia Nueva. Se analizan específicamente sus señalamientos sobre la objetividad en la ciencia, la relación entre ciencia y sociedad, el rol del científico en la sociedad y la relación entre actividad científica y política. Se destaca la originalidad que presenta el debate en términos de la relación entre epistemología, ciencia, política y procesos sociales, que permite repensar los aportes de los autores en torno a la actualidad del Pensamiento Latinoamericano en Ciencia, Tecnología y Desarrollo (PLACTED) y del campo CTS.

\section{Introducción}

En el año 1971, la revista Ciencia Nueva publica un reportaje a Gregorio Klimovsky, que inicia una polémica con Oscar Varsavsky, Jorge Schvarzer, Manuel Sadosky, Conrado Eggers Lan, Thomas Moro Simpson y Rolando García. El libro Ciencia e Ideología. Aportes polémicos (1975), recopila parte de los artículos publicados en dicha revista en relación con esta polémica.

Este tipo de debates se dan en pleno desarrollo de lo que se dio en llamar el Pensamiento Latinoamericano en Ciencia, Tecnología y DesarroIlo (PLACTED), un movimiento surgido a fines de la década de 1960 integrado por un conjunto de voceros provenientes de diferentes ámbitos (universidades, organismos gubernamentales, empresas, etc.) preocupados por la relación entre la ciencia, la tecnología y las problemáticas sociales. Si bien estos voceros presentan posiciones heterogéneas, "un elemento común los aunaba: el carácter político de sus preocupaciones, la función política de su discurso. Más allá de las diferencias ideológicas, no parece arriesgado afirmar que el contexto de su pensar era la actividad militante." (Dagnino et al., 1996: 30). Varsavsky y Rolando García representaban las visiones más radicales dentro de esta corriente de pensamiento y consideraban que era necesaria una transformación social revolucionaria para lograr el desarrollo de los países latinoamericanos.

Por otro lado, otros intelectuales como Klimovsky y Moro Simpson, si bien no fueron parte de esta 
| Ciencia, Tecnología y Política | Año 4 | N7 | e067 | Noviembre 2021 | ISSN 2618-3188 | www.revistas.un|p.edu.ar/CTyP |

corriente de pensamiento, tenían una mirada crítica de algunos aspectos de la visión optimista de la ciencia y la tecnología que prevalecía desde las décadas del '50 y '60 del Siglo XX, que proponía que para alcanzar el desarrollo bastaba con copiar el modelo seguido por los países centrales.

El presente trabajo recupera las posiciones asumidas por Gregorio Klimovsky, Oscar Varsavsky y Thomas Moro Simpson en el debate sobre ciencia e ideología. Específicamente sus señalamientos sobre la objetividad en la ciencia, la relación entre ciencia y sociedad, el rol del científico en la sociedad, y la relación entre actividad científica y política.

\section{La revista Ciencia Nueva}

La Revista Ciencia Nueva, dirigida por Ricardo Ferraro y publicada entre los años 1970 y 1974, fue un espacio desde donde se articularon los principales debates de la época en torno a la ciencia, tecnología y política. Quienes escribieron en ella (personalidades como Manuel Sadosky, José Babini, Mario Bunge, Jorge Sábato, Gregorio Klimovsky, Julio Moreno, Manuel Risueño, Darcy Ribeiro, Eduardo De Robertis, Rolando García, Oscar Varsavsky, Conrado Eggers Lan, Jorge Schvarzer, Thomas Moro Simpson, Amílcar Herrera, Jorge Katz y Enrique Oteiza, entre otros), provenían de diferentes disciplinas. Las temáticas tratadas versaban sobre la teoría de la dependencia, subdesarrollo, autonomía científica, la objetividad en la ciencia, el rol de la universidad. Las posiciones que asumieron estos autores contribuyeron en su época a la construcción de un imaginario sobre el lugar de la ciencia y la tecnología en la sociedad, y aportan elementos para las reflexiones actuales del campo CTS.

Un aspecto a destacar es que si bien en todos estos debates que reflejó ciencia Nueva hay grados de heterogeneidad y posiciones opues- tas, los mismos mantienen una cercanía con el PLACTED en el compromiso de la transformación social, la liberación latinoamericana, el cuestionamiento al modelo político y científico vigente y una fuerte oposición al imperialismo y la injerencia de políticas científicas extranjeras (Céspedes, 2019). Así se entienden los debates que se despliegan a lo largo de las diferentes publicaciones, como el que aquí se tratará.

\section{Gregorio Klimovsky: La ideología no afecta la objetividad}

La polémica que se desarrolla a lo largo de la revista, se inicia con una entrevista a Klimovsky, quien afirma que la ideología "interviene en la ciencia, sin que eso (...) afecte su objetividad, porque no se trata de la misma entidad que otros denominan con esas palabras" (Klimovsky et al., 1975: 13). Sobre esta base, presenta cuatro formas de concebir la ideología.

Por un lado, ideología como marco teórico o conceptual desde el cual se posiciona el científico para la elaboración de una teoría o investigación. Este encuadre es un aspecto legítimo y natural para el proceso de investigación, puesto que es planteado explícitamente por el científico. No afecta, así, la objetividad de la ciencia.

Una segunda posibilidad es la Ideología desde el punto de vista de la sociología del conocimiento. Se aplica a la persona que, de acuerdo a la influencia de su contexto socio-histórico, desarrolla una investigación desde una determinada perspectiva implícita. En este caso, la cuestión que atenta contra la objetividad, sería la distorsión que el científico, por estar ubicado en una clase social determinada, puede ejercer sobre los problemas relevantes en que debe trabajar; puesto que los problemas relevantes para un grupo social pueden no ser relevantes para otra clase social. Para Klimovsky, esto puede solucionarse con adiestramiento y crítica en el método científico mediante el cual el científico "pueda 
| Ciencia, Tecnología y Política | Año 4 | No7 | e067 | Noviembre 2021 | ISSN 2618-3188 | www.revistas.un|p.edu.ar/CTyP |

darse cuenta de su propia información" (Klimovsky et al., 1975: 16).

Una tercera perspectiva es la ldeología en virtud de intereses espurios, en la cual los científicos producen conocimientos y líneas de investigación por razones de conveniencia personal. En este caso, al actuar en función de intereses personales atenta contra la verdadera práctica científica.

Finalmente, la cuarta alternativa es la ideología como escasez o imposibilidad de información. Acá, el problema ideológico se produce cuando una región no logra el desarrollo (científico) porque no ha recibido la información relevante y necesaria, que sí llega a otra región en el mismo momento histórico.

Por otro lado, Klimovsky, siguiendo la concepción heredada del Círculo de Viena ${ }^{1}$ asume la distinción tradicional de los contextos de la actividad científica: contexto de descubrimiento, contexto de justificación, contexto de aplicación. Respecto del contexto de justificación, afirma que "Ios factores ideológicos que aparecen son pocos y escasamente molestos" (Klimovsky et al., 1975: 22). Considerando que el científico contrasta las hipótesis desde un marco conceptual aceptado (según el primer sentido de ideología), "no hay ningún inconveniente en cuanto al valor objetivo del conocimiento obtenido" (Klimovsky, et al., 1975: 24). Sin embargo, el autor indica un posible problema denominado "autoalimentación de la teoría", esto implica un círculo vicioso: el científico utiliza su propio marco conceptual para evaluar sus resultados que son propuestos por su propia teoría. De este modo, cae en una incomprensión total del método científico. Por el contrario, es necesario una aplicación adecuada del método para no caer en el problema de la ideología en el tercer sentido. Estos problemas se resuelven fácilmente en la medida en que "la crítica epistemológica puede eliminar errores metodológicos como los que acabamos de describir" (Klimovsky, et al.,1975: 27).

Para Klimovsky, el problema central aparece cuando la ideología interviene en el contexto de descubrimiento que puede obstaculizar el desarrollo de la ciencia de un país. La hipótesis que propone un científico "puede venir sugerida por investigaciones análogas que se han hecho en otro lugar, por modas, por apreciaciones acerca del alcance y valor de un tipo de estudio (...), o por un tipo de experiencia que no es típica de nuestro medio" (Klimovsky et al., 1975: 28).

En el contexto de aplicación, Klimovsky identifica uno de los peores problemas ideológicos en el sentido espurio. Analiza la forma en que los intereses de la política extranjera limitan y direccionan las políticas tecnológicas para el desarrollo del país. El problema es la "falta de información o la falta de adecuación de técnicas del extranjero a las necesidades locales" (Klimovsky et al., 1975: 30), lo que ha dificultado, en el caso argentino, lograr algo útil.

En la segunda parte de la entrevista, el epistemólogo coincide en la necesidad de un cambio social que "va a requerir técnicos y científicos para organizar y llevar a cabo los nuevos programas" (Klimovsky et al., 1975: 31). Bajo la responsabilidad del cambio social, señala tres actividades fundamentales a llevar a cabo: ser "vigilantes científicos" que puedan denunciar los errores sociales y tecnológicos que frenan el desarrollo del país;

1 El Círculo de Viena fue una organización de librepensadores que entre 1922 y 1936 se nuclearon alrededor de Moritz Schlick, titular de la cátedra de filosofía de las ciencias inductivas en la Universidad de Viena (Austria). Hans Hahn, Otto Neurath y Rudolf Carnap, integrantes de este grupo, publican en 1929 el Manifiesto del Círculo de Viena titulado "La concepción científica del mundo" considerado el inicio de la epistemología o filosofía de la ciencia. En términos generales proponían la construcción de un lenguaje universal de la ciencia, libre de toda pretensión metafísica, fundamentado en el análisis lógico, con el fin de unificar la ciencia y comunicarla a la sociedad. Las ideas originales fueron sufriendo modificaciones que culminaron en una versión final denominada concepción heredada que influyó fuertemente en los desarrollos epistemológicos posteriores. 
| Ciencia, Tecnología y Política | Año 4 | N7 | e067 | Noviembre 2021 | ISSN 2618-3188 | www.revistas.un|p.edu.ar/CTyP |

realizar estudios sobre las condiciones de posibilidad para la implementación de los programas sociales, económicos, tecnológicos, educativos que requiere el cambio social, dejando los "slogans" políticos y brindando verdaderas soluciones; y, resolver los problemas luego del cambio social. Rechaza la idea de importar técnicos y científicos extranjeros, dado que, por sus concepciones políticas y sociales ajenas a nuestra realidad, se recae en problemas ideológicos (en el segundo y tercer sentido antes señalado). Para Klimovsky el científico debe dividir su trabajo entre la investigación en ciencia básica y en el análisis de los problemas nacionales existentes: "qué hacer con el problema antes, después y durante el cambio" (Klimovsky et al., 1975: 35).

Finalmente, afirma que "una cosa es la acción política y otra la acción científica. Son conceptos y tareas que no deben confundirse" (Klimovsky et al., 1975: 36). El científico puede comprometerse con la política en cuanto persona, pero no puede mezclar la actividad científica con la política, puesto que contamina y no resulta positivo para el cambio del país. Respecto de hacer ciencia nacional, cree que es un error y un peligro pensar métodos especiales para el diseño de investigaciones que respondan a la idiosincrasia y al "ser nacional" tal como ocurrió con los delirios de Hitler defendiendo una "ciencia alemana" (Klimovsky et al., 1975: 37). La ciencia nacional es "toma de conciencia acerca de nuestros problemas argentinos, el estudio de técnicas para resolverlos, el detectar hipótesis y teorías que puedan auxiliarnos, el ordenamiento racional de nuestra enseñanza, etc. (Klimovsky et al., 1975: 37).

\section{Oscar Varsavsky: La ciencia es ideológica}

Por su parte, Oscar Varsavsky en Ideología y Verdad, sostiene que "la ciencia actual está saturada de ideología a todo nivel, como cualquier otra actividad social, que ella es muy visible en algunos niveles (usos de la ciencia) y en otros está más disimulada" (Klimovsky et al., 1975: 42). Rechaza la clasificación de ideología de Klimovsky, argumentando que son definiciones abstractas, y que esta clasificación encierra un "vicio ideológico" que limita y anula todo tipo de discusión. También se opone a la distinción de los contextos de la actividad científica, puesto que esta diferenciación es funcional para quienes pretenden reducir la ciencia al análisis proposicional (análisis lógico y lingüístico), sin importar el surgimiento de hipótesis y su aplicación tecnológica (Klimovsky et al., 1975: 43).

A propósito de su crítica a la epistemología propuesta por el positivismo lógico, en Ciencia, política y cientificismo (Varsavsky, 1969) ya señalaba que hay una dimensión que no es considerada para el análisis de la ciencia y de las proposiciones científicas (leyes, teorías científicas). Se trata del criterio de importancia que no puede ignorarse si se quiere pensar una ciencia en relación con los problemas sociales (Varsavsky, 1969: 51).

Varsavsky cambia la perspectiva de Klimovsky, señala que el aspecto ideológico en la ciencia aparece en la propia discusión sobre cuáles son los problemas que debe abordar la ciencia, "estos problemas pueden plantearse en abstracto 0 referidos a la ciencia actual. Centrar la discusión en las características ideales que la ciencia debería o podría tener, es un acto ideológico" (Klimovsky et al., 1975: 41). La ideología aparece en la ciencia en la medida en que se discuta cuál es el valor de importancia que se otorga a los problemas que debe abordar la ciencia para el desarrollo y transformación de la sociedad:

El problema que está en juego aquí es la transformación de esta sociedad en otra. Se trata entonces de ver si hay una manera de hacer ciencia que ayuda a esta transformación y otra que la dificulta, y hasta donde llegan estas diferencias. Eso es lo que a mí me interesa usar para definir 
ideología en ciencia (Klimovsky et al., 1975: 42).

Para Varsavsky, el criterio de importancia implica otorgar prioridad a los problemas locales para que posibiliten un cambio social y brinden autonomía científica. En este sentido, en Bases para una política nacional de Tecnología y Ciencia (Klimovsky et al., 1975: 52-57), afirma que, si aceptamos la concepción de desarrollo lineal, en el cual los países que están en un nivel superior funcionan como modelos a seguir para los países dependientes, el criterio de importancia viene dado de afuera, produciendo "dependencia cultural" (Klimovsky et al., 1975: 52). En el proceso de construcción del conocimiento, la investigación tecnológica y científica debe corresponder a las necesidades populares. De este modo se configura un "estilo tecnológico" (Varsavsky, 1974) propio acorde a la realidad social del momento. En el artículo Ciencia y estilos de desarrollo (1971) afirma: "la ciencia es parte de la cultura y como tal de la sociedad, lo que nos lleva a tener que elegir y definir qué sociedad pretendemos antes de saber qué ciencia haremos" (Varsavsky, 1971: 38). Discutir qué tipo de ciencia obstruye o construye un modelo social que se elige construir, es pensar en términos ideológicos. Varsavsky apunta explícitamente contra el "estilo continuista" que considera a la sociedad norteamericana como modelo social.

¿Es entonces la ciencia objetiva? En respuesta, Klimovsky asume una posición de resguardar la objetividad científica, libre de toda ideología, a través de "buenas metodologías", acentuando el carácter de veracidad, o no, de las hipótesis. Varsavsky asume, en una postura opuesta, un posicionamiento plenamente ideológico, es decir, la necesidad de plantear una política de ciencia y tecnología a partir de un Proyecto Nacional.

Respecto de la actividad del científico, Varsavsky cuestiona la pasividad que le otorga Klimovsky a los científicos aun cuando éste le asigne cierta militancia y compromiso por el cambio social. Al reducir la actividad científica en el contexto de justificación, al científico solo le resta ser un técnico de control de calidad determinando la verdad o falsedad de las hipótesis. Varsavsky propone que

la misión del científico rebelde es estudiar con toda seriedad y usando todas las armas de la ciencia, los problemas del cambio de sistema social, en todas las etapas y en todos sus aspectos, teóricos y práctico. Esto es hacer ciencia politizada (Varsavsky, 1969: 25).

Por otro lado, hay un punto común en el distanciamiento con los países centrales. En este sentido, Klimovsky señala el problema de no ajustar la ciencia a la realidad nacional y acusa del peligro de importar, por ejemplo, un modelo socialista; mientras que Varsavsky, afirma que no es conveniente que los jóvenes científicos estudien en el exterior porque su falta de madurez permite que sean susceptibles de ser "compradores" del canon de la ciencia universal, la de los países dominantes, "así es como vuelven al país como misioneros de una política científica extranjera, muy prestigiada, pero que no sirve a la Liberación sino a la dependencia" (Klimovsky et al., 1975: 56).

Otro contrapunto es la especial vinculación que traza Varsavsky entre ciencia y política, "la actitud del científico tiene que ser ideológica y constructiva (...) la ideología es elección y deben elegirse tanto los problemas a estudiar como los métodos a aplicar y la organización social de los científicos para ayudar el proceso de cambio" (Varsavsky, 1971: 39). Debemos recordar que años antes, en Ciencia política y cientificismo (1969), definía esta relación desde la idea de "ciencia politizada"2. El científico ya no como aquellos que reproducen lógicas científicas funcionales a modelos ortodoxos e imperialistas, sino como aquel que estudia

2 Para un análisis más detallado sobre la relación ética, ciencia y política en experiencias en Argentina, ver Bilmes, et al., 2018. 
con toda seriedad los conceptos de la ciencia en función de los problemas sociales para luego resolverlos.

\section{Las críticas de Thomas Moro Simpson}

Thomas Moro Simpson en Irracionalidad, ideología y objetividad (Klimovsky et al., 1975: 79108), arroja un arsenal crítico a las posturas antes mencionadas. Señala que el debate entre ciencia e ideología es surrealista, y que resulta más interesante abordar los puntos en común que las diferencias. Para él, Varsavsky no solo se aleja del sentido marxista de ideología como falsa conciencia, sino que además no define explícitamente qué entiende por ideología (porque considera que es caer en la misma ideología). En todo caso, es posible inferir que lo ideológico se entiende como aquello que favorece o dificulta el desarrollo de un país en los términos en que él (por Varsavsky) lo entiende de manera más adecuada; y aquello que no contribuye al desarrollo, lo dificulta. De este modo, "el hecho de que las ideas de GK [Gregorio Klimovsky] no tengan la virtud de acelerar el proceso revolucionario no las convierte en falsedades" (Klimovsky et al., 1975: 82).

Respecto de la objetividad, Moro Simpson le cuestiona a Varsavsky tergiversar semánticamente el concepto de objetividad. Mientras que Klimovsky define objetividad como propiedad de las teorías científicas para el contexto de justificación, Varsavsky sostiene que no hay objetividad desde el punto de vista del criterio de importancia. No advierte "que está usando la palabra 'objetividad' con otro sentido, tan legítimo como el anterior pero diferente" (Klimovsky et al., 1975: 83). Coincide en el análisis de que no existe imparcialidad para la determinación de las políticas científicas, pero esto no significa que no haya objetividad en sentido gnoseológico.

En otro ataque a Varsavsky, lo sitúa en una mala comprensión del punto de partida para la actividad científica. Según él, Varsavsky se limita a criticar una imagen de ciencia centrada en la justificación de hipótesis y en el carácter pasivo del científico como "control de calidad". Todo esto es "un invento de Varsavsky: la ciencia se caracteriza por descubrir problemas y buscar respuestas cada vez más amplias y precisas" (Klimovsky et al., 1975: 96).

Finalmente, señala que la mala predisposición de Varsavsky contra el positivismo lógico, lo conduce a no considerar otros posicionamientos de los positivistas como Philipp Frank que, por ejemplo, asumen un fundamento ético para el criterio de aceptabilidad.

\section{Reflexiones}

Un aspecto común que comparten los autores mencionados refiere a la necesidad del cambio social pensado desde el contexto social. Sin embargo, en el análisis de los aspectos que discuten estos pensadores, se puede advertir algunas diferencias sustanciales respecto a la incidencia de la ideología en la ciencia. Ha quedado expresado que para Klimovsky la ideología en ciencia es un problema que atenta contra la objetividad científica, mientras que para Varsavsky la ciencia se define ideológicamente. Moro Simpson advierte de lo superfluo que es esta discusión, y que al parecer no conduce a ningún puerto.

Respecto a la objetividad, mientras que Klimovsky sostiene que habría una posición objetiva dada por el proceso de contrastación de hipótesis, en Varsavsky y Moro Simpson se asume que hay un posicionamiento ideológico en las políticas de CyT y por tanto en los procesos de investigación que se ejecutan.

Respecto del rol del científico, los autores confluyen en la necesidad de contribuir a la resolución de problemas para el cambio social. La postura 
| Ciencia, Tecnología y Política | Año 4 | No7 | e067 | Noviembre 2021 | ISSN 2618-3188 | www.revistas.un|p.edu.ar/CTyP |

de Varsavsky es que deben hacer una ciencia politizada, usar la ciencia para estudiar los problemas del cambio de sistema social. En este punto se advierte cierta tensión en Klimovsky, ya que mientras que rechaza la posición de Varsavsky, sostiene que el cambio social requiere de técnicos y científicos para desarrollar los nuevos programas (Klimovsky et al, 1975: 31), es decir, una mirada distinta a la perspectiva más politizada, pero que se esfuerza por articular ciencia y cambio social.

Finalmente, señalamos la originalidad del debate en la década del '70, en la que es posible unificar epistemología, ciencia, política y procesos sociales, que es uno de los aportes que se transfieren para una epistemología política de CyT. Asimismo, dos cuestiones nos parecen interesantes de destacar para repensarlas en términos de la actualidad del PLACTED.

En primer término, llama la atención en el debate la recuperación que hacen estos autores del positivismo lógico en relación con la valoración de la ciencia. Klimovsky y Varsavsky, y advertida en cierto sentido por Moro Simpson, reconstruyen una línea de análisis que deja de lado o ignora ciertos aspectos que el positivismo lógico ha señalado al respecto. Si revisamos el Manifiesto del Círculo de Viena (Neurath, et al., 2002), podemos señalar que este grupo ya asumía fuertes compromisos políticos para la ciencia. Las investigaciones de las últimas décadas, y que por cierto son posteriores a este debate (Friedman, 1999; Reisch, 2009; Gómez, 2014), revisan la mirada que la historia oficial ha construido sobre las tesis positivistas, es decir que lejos de asimilar una visión neutral de la ciencia, proponían una ciencia en relación con los problemas de la época. Una posible explicación de porqué estas cuestiones no son tenidas en cuenta podría tener que ver con el hecho de que luego de la segunda guerra mundial, las presiones políticas fueron tales (como por ejemplo el macartismo en Esta- dos Unidos), que obligó a los pensadores positivistas a despojarse de sus objetivos políticos y afiliaciones a la izquierda marxista. Así, es que se produce cierto giro hacia la despolitización y neutralidad científica, y la tarea de la ciencia queda reducida al análisis lógico e interpretación de teorías. Esta fotografía de época es la que denominó Putnam en 1962 como concepción heredada, la versión final del Círculo de Viena (Putnam, 1989). Las publicaciones que siguieron a este acontecimiento, como las de Ayer (1985), Giere (1988), Putnam (1989), Salmon et al. (1999), citados en Araujo y Medina (2014), reproducen una imagen tergiversada o incompleta de la propuesta de ciencia del positivismo lógico. Suponemos que esta es la versión a la que se refieren estos pensadores en el intento de redefinir a la ciencia para el nuevo contexto argentino. Si bien Varsavsky no proviene del ámbito epistemológico, resulta interesante señalar estos aspectos que generan nuevos interrogantes sobre la relación entre el grupo de pensadores comprometidos con una ciencia nacional, con el positivismo lógico.

En segundo término, el debate se vuelve ameno en la medida en que asimilamos que hay puntos en común entre Klimovsky y Varsavsky en la idea de una ciencia contextualizada y en relación con Ios problemas sociales en vistas a una transformación social; en otras palabras, la ciencia no es algo que pueda considerarse en abstracto, como si fuesen verdades absolutas siguiendo lo que impone la "ciencia universal". En este sentido siguen quedando preguntas abiertas para el debate, entre otras, ¿cómo y quiénes definen el rumbo de la ciencia?, ¿alcanza con contar con científicos politizados o revolucionarios? A propósito de estas preguntas, podemos señalar, siguiendo a Dussel (1993) y la perspectiva decolonial, que en todo caso el desafío es construir espacios pluriversales, con una dimensión epistemológica desde el sur. Espacios en los que se integren los saberes alternativos, y, siguiendo el legado de 
esta época, avanzar hacia la construcción de una ciencia y una tecnología nacionales con autonomía, soberanía e independencia para resolver las necesidades de nuestra sociedad.

Agradecimiento: a Santiago Liaudat por la motivación para reelaborar y presentar este trabajo.

\section{Bibliografía}

Araujo, C. I. y Medina C. G. (2014). Acerca de la polémica Neurath-Horkheimer: ciencia y política. Diánoia, 59 (72), 113-129.

Bilmes, G., Carrera J., Andrini L., Liaudat, S. (2018). Ética, ciencia y compromiso político. Opciones y alternativas desarrolladas por científicos/as sensibles a los problemas sociales. En M. G. Ortúzar (Comp.). Ética, ciencia y política: hacia un paradigma ético integral en investigación. Universidad Nacional de La Plata. https://www.libros.fahce. unlp.edu.ar/index.php/libros/ catalog/book/133

Céspedes, L. (2019). La revista argentina ciencia nueva (1970-1974): Análisis de contenidos, recursos gráficos, publicidad y públicos. Perspectivas de la Comunicación 12(1), 281-313.

Dagnino R.; Thomas H.; Davyt, A. (1996). El pensamiento en ciencia, tecnología y sociedad en Latinoamérica: una interpretación política de su trayectoria. Redes 7(3), 1351.

Dussel, E. (1993). Europa, modernidad y eurocentrismo. En E. Lander (comp.), La colonialidad del saber: eurocentrismo y ciencias sociales. Perspectivas latinoamericanas. CLACSO.

Friedman, M. (1999). Reconsidering Logical Positivism. Cambridge University Press.

Gómez, R. (2014). La dimensión valorativa de las ciencias. Hacia una filosofía política. Universidad Nacional de Quilmes.

Klimovsky, G.; Varsavsky, O.; Schvarzer, J.; Sadosky, M.; Eggers Lan, C.; Moro Simpson, T.; García, R. (1975). Ciencia e ideología. Aportes polémicos.

\section{Ediciones CIENCIA NUEVA.}

Neurath, O., Carnap, R., Hahn, H. (2002). La concepción científica del mundo: el Círculo de Viena. Redes, 9 (18), pp.5-149.

Putnam, H. (1989). Lo que las teorías no son. En L. Olivé y A. R. Pérez Ransanz (comps.), Filosofía de la ciencia: teoría y observación (pp. 312-329). Siglo XXI.

Reisch, G. A. (2009). Cómo la Guerra Fría transformó la filosofía de la ciencia. Hacia las heladas laderas de la lógica. Universidad Nacional de Quilmes.

Varsavsky, O. (1969). Ciencia, política y cientificismo. CEAL.

Varsavsky, O. (1971). Ciencia y estilos de desarroIlo. Ciencia Nueva, 2 (13), 38-39.

Varsavsky, O. (1974). Estilos tecnológicos. Ediciones Periferia. 